\title{
Semántica creciente y decreciente a partir de conocimientos previos en la redacción científica
}

\section{Increasing and decreasing semantics based on previous knowledge in scientific writing}

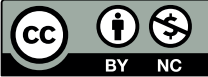

\author{
Francisco Villao Villacrés ${ }^{1}$ \\ fvillaov@ulvr.edu.ec \\ Ruth Garofalo García ${ }^{1}$ \\ rgarofalog@ulvr.edu.ec
}

Recibido: 1/07/2017, Aceptado: 1/09/2017

\begin{abstract}
RESUMEN
El presente estudio de investigación se fundamenta en el desarrollo de las habilidades para la redacción de los textos científicos, lo que exige un dominio semántico que haga posible la distribución del proceso comprensivo, para la utilización correcta de signos lingüísticos a través de palabras, expresiones y oraciones. Para esta investigación se aplicó el método cualicuantitativo integrado por los estudiantes de quinto, sexto y séptimo semestre de la Carrera de Inglés de la Universidad Laica "Vicente Rocafuerte" de la ciudad de Guayaquil, con el propósito de identificar las dificultades que los estudiantes de educación superior presentan al expresar los conocimientos en redacción científica. Mediante el análisis de sus antecedentes lexicales y con el afán de proponer soluciones didácticas en el ejercicio de su profesión y mejorar la calidad de su producción escrita, se aplicaron un test de diagnóstico y según criterios de evaluación dio como resultado la baja calidad de escritura utilizada por los entes en estudio; detectándose que los conocimientos previos no están presentes en el proceso evaluativo. Se pretende sembrar en los estudiantes un tipo de investigación de calidad haciendo uso del aprendizaje significativo, el mismo que tiene como objetivo detectar las falencias en la escritura de redacciones, párrafos, ensayos, etc. Con el afán de contribuir al desarrollo de la escritura en un país en vías de desarrollo.
\end{abstract}

Palabras clave: aprehensión- cognitivo- conocimientos previos

${ }^{1}$ Docente de la Facultad Ciencias de la Educación - Mención Inglés Universidad Laica Vicente Rocafuerte de Guayaquil. Ecuador

Revista Científica Ciencia y Tecnología Vol 17 No 16

II Jornada de investigación págs. 23-33

http://cienciaytecnologia.uteg.edu.ec 
Revista Ciencia \& Tecnología No. 16, 31 de octubre de 2017 ISSN impreso: 1390 - 6321

\section{Jornada de Investigación Universidad Tecnológica Empresarial de Guayaquil}

\begin{abstract}
The writing of the scientific texts requires the author's part, a semantic domain that is possible the distribution of the comprehensive process, for the correct application of linguistic signs through words, expressions and sentences and the development of skills and abilities in writing. In order to justify these principles, the objective is to demonstrate the difficulties that the students of higher education present to demonstrate the knowledge in scientific writing. By means of the analysis of his lexical antecedents with the desire of the proponent didactic solutions in the exercise of his profession. The morphological expression to drift what it does is to increase the gap in the comprehensive writing, once the text is formed, it will be necessary to know the cognitive level of that reality expressed as much as the author as the issuer. If students dominate cognition and use idiomatic segments, they use prepositions and conjunctions for paragraphs that complement their morphological structure. A qualitative and quantitative fieldwork will be carried out with fifth, sixth and seventh semester students from the Language School of the Vicente Rocafuerte Laica University in the city of Guayaquil.
\end{abstract}

Keywords: cognitive apprehension - previous knowledge

\title{
Introducción
}

La divulgación de la ciencia mediante artículos de investigación científica en revistas indexadas ha dejado de ser una opción para muchos docentes y estudiantes universitarios lo cual se ha convertido en una necesidad para quienes ejercen la investigación; sin embargo, existe el desconocimiento de producción y publicación científica. Por otra parte, los estudiantes no están preparados para la adaptación a este género de carácter científico en el entorno de sus aprendizajes. Estos problemas se observan en los estudiantes de la Facultad de Educación en la Carrera de Inglés de la universidad laica Vicente Rocafuerte de Guayaquil, debido a que muchos de ellos no han recibido una instrucción formal en escritura científica y por ende revelan serias limitaciones en las redacciones por desconocimientos de estructuras semánticas y sintácticas, presentando problemas generales de la escritura, impidiendo desarrollar con normalidad los ensayos y producciones científicas.

El desafío para muchos estudiantes universitarios por lograr obtener un espacio en al ámbito investigativo y que sus escritos sean publicados en revistas indexadas ha permitido incrementar su curiosidad e interés hacia la búsqueda de información y mejorar sus habilidades escritas; sin embargo, existe una brecha que se ha ampliado y se agiganta a diario debido a los conocimientos básicos adquiridos desde su formación escolar en relación con las destrezas que los estudiantes adquieren para la redacción de ensayos o artículos. Desde el punto de vista analítico los estudiantes al ingresar a las universidades no pueden realizar trabajos escritos designados por sus docentes; es evidente la carencia de fundamentaciones teóricas y la correlación entre párrafos, la incorrecta ubicación de signos de puntuación, errores gramaticales y de semántica.

De acuerdo a la constitución de la República del Ecuador (Asamblea Nacional, 2008), en su artículo 287 promueve "la generación y producción de conocimiento, fomenta la investigación científica y tecnológica y asegura la difusión y el acceso a los conocimientos científicos y tecnológicos." Las universidades a nivel nacional en la actualidad demandan exigencias en sus docentes en realizar actividades relacionadas en la investigación y fomentar en sus estudiantes el interés hacia la producción 


\section{Jornada de Investigación Universidad Tecnológica Empresarial de Guayaquil}

científica mediante ensayos o artículos. Por lo tanto, es imprescindible desarrollar en el estudiante habilidades para lecto escritura, acompañado desde un aprendizaje significativo a través del conocimiento previo, orientando hacia la indagación y el descubrimiento por las cosas para su investigación para luego fundamentarlo de una manera teórica y cualitativa a través de sus escritos.

\section{Importancia del problema de la investigación}

La presente investigación acerca del dominio lexical estudiantil para la producción científica en los estudiantes de la Carrera de Inglés es conveniente porque está encaminada a la solución de un problema de carácter educativo. El propósito es difundir la cultura científica en los estudiantes y contribuir hacia el desarrollo de la habilidad escrita que a menudo se presentan muchas falencias al momento de escribir un ensayo o artículo académico. Esta investigación implica en resolver un problema que se presenta a diario en los estudiantes en las redacciones de ensayos a través de estrategias didácticas y una orientación necesaria, logrando optimizar sus habilidades escritas el desarrollo de redacciones científicas.

Tiene un valor teórico debido a que se fundamentan de fuentes caudales bibliográficos de varios autores que han favorecido hacia el desarrollo científico en la sociedad, enriqueciendo a su vez hacia el desarrollo del perfil de egresado como investigadores y redactores científicos e incrementando considerablemente el nivel académico y desempeño profesional de los futuros docentes de inglés mediante el hábito hacia la lectura y su producción científica.

Actualmente la ciencia debe ser expresada a través de Artículos de Investigación Científica en revistas indexadas, convirtiéndose en una opción primordial en el desarrollo de habilidades escritas en los estudiantes que forman parte de un país en vías de desarrollo, lo cual se ha transformado en una necesidad para quienes inician o ejercen la investigación. Esto se debe a que el aumento de las publicaciones indexadas juega un papel esencial, en los procesos de reasignación en los sistemas de acreditación de las instituciones de Educación Superior.

(El Mercurio, 2015), menciona que Senescyt ha detectado que el 30 por ciento de estudiantes de posgrado no han podido culminar sus estudios por problemas de escribir, de igual manera se detecta en estudiantes de pregrado que desertan con frecuencia por los altos niveles y exigencias de búsqueda de información donde los estudiantes deben recurrir a bibliotecas locales o virtuales, sin embargo este escenario no se evidencia a diario, puesto que no tienen hábitos de lecturas y están acostumbrados a leer resúmenes o solo ideas necesarias para completar su trabajo escrito y con alto grado de deficiencia en ortografía, coherencia y cohesión a la hora de escribir. A través del hábito hacia la lectura, se fortalece la destreza de la escritura e incrementa su vocabulario; reconoce las pausas de cada oración y coloca correctamente los signos de puntuación.

La procedencia de un Sistema Educativo con deficiencias en la enseñanza de la expresión oral y escrita se ve evidenciada en los trabajos, redacciones, ensayos, etc., que se reciben de los alumnos de la Carrera de Inglés y con mucha frecuencia se observan errores que se podrían encontrar en un escrito elaborado por un estudiante de educación básica media.

Otro factor que se ha podido constatar es que estas falencias se vienen arrastrando desde la escuela y colegio y se agravan por la falta de hábitos de lectura y el mal uso 


\section{Jornada de Investigación Universidad Tecnológica Empresarial de Guayaquil}

de la tecnología. Los jóvenes tienen ahora la costumbre de acortar las palabras cuando escriben en el teléfono celular y que muchos de estos aparatos corrigen en forma automática los errores ortográficos.

Estas deficiencias en redacción y ortografía con lo que los jóvenes llegan a la enseñanza superior, resulta dificultoso implementar estrategias metodológicas de des-aprendizaje, lo que ocasiona que muchos de los alumnos no puedan redactar un ensayo sencillo; escriben un par de líneas, no saben concatenar ideas; la ortografía es poco aceptable.

Tienen una falencia total de estrategias de redacción, la cohesión de ideas, la puntuación, colocar tildes entre otros.

Según la prensa (La Nación, 2017), mediante un estudio de campo afirma que "Los niños cuyos padres manejan un léxico adecuado y que tienen maestros que les fomentan la lectura dominan un vocabulario de unas 1.200 palabras. Si ocurre lo contrario, el léxico que manejan puede llegar máximo a 200 palabras." Por lo tanto, se debería implementar en las universidades un rígido sistema de lectura y escritura en los primeros semestres, lo cual daría resultados favorecedores que servirían de aporte a la escritura de documentos científicos.

La falta del hábito de la lectura se evidencia en los estudiantes de la Carrera de Inglés, la mayoría de los alumnos se resisten a leer; buscan la conformidad del material impartido por el docente, la lectura que ellos realizan es rápida y poco crítica. Se observa también que las visitas que realizan los estudiantes a la biblioteca son poco frecuentes y escasas; por lo tanto, el interés por investigar o leer artículos de acuerdo a las asignaturas es insuficiente. De acuerdo a los trabajos realizados por los estudiantes existe poco criterio en realizar investigaciones de forma empírica, basándose en las experiencias obtenidas en vinculación o prácticas docentes.

(Feldman, Anderson, \& Mangurian, 2001) revisan una serie de problemas, a los que se enfrentan los estudiantes que ingresan a la Universidad en relación a la escritura, la lógica, incongruencias estilísticas relativas a la disciplina de la redacción y está envuelta en una serie de debilidades mecánicas (gramática y vocabulario), lo cual ha ocasionado problemas para obtener un léxico nutrido. La publicación de artículos en revistas especializadas e indexadas, no solo se ha transformado en una práctica clave en la divulgación del conocimiento científico, sino también en un indicador sustancial en el desarrollo de habilidades de redacción en los estudiantes de la Carrera de Inglés. Es importante para los docentes investigadores primero descubrir los problemas de redacción en los estudiantes con relación a la lengua materna, para fortalecer sus habilidades de comprensión lectora, lo cual llevaría a mejorar la calidad de la redacción científica.

Otra de las causas que podrían estar ocasionando la decadencia en la escritura científica es el uso inadecuado de la tecnología; no obstante, muchos estudiantes consideran esto como un método que les permite actuar con rapidez y comodidad para la redacción a través de programas informáticos como: Excel, Word, PowerPoint, etc. Estos utilitarios poseen correctores automáticos que permiten combinar palabras para formar oraciones semánticamente organizadas; sin embargo, al iniciar la interacción de la computadora con los usuarios suceden una serie de situaciones ilógicas, debido a que los ordenadores producen secuencia de textos correctamente combinados; pero, a su vez no presentan relación con las estructuras comunicativas. 


\section{Jornada de Investigación Universidad Tecnológica Empresarial de Guayaquil}

A pesar de las múltiples reglas ortográficas que Microsoft Word ofrece, los resultados en la redacción carecen de un componente semántico que permita comprender al lector el elemento significativo de transferencia (Searle, 2014).

\section{Incoherencia de contenidos entre ideas principales y secundarias}

Los problemas que los estudiantes encuentran al desarrollar un escrito académico, está en que al tiempo de interpretar un texto leído presenta incongruencias en el resultado de su trabajo. Debido a la falta de hábitos de lectura, los estudiantes no ubican la idea principal; por lo tanto, el hábito de una lectura minuciosa baja el nivel de redacción científica, dificultando la ubicación, jerarquización y el concepto fundamental de una información, (Insirillo, Gonzalez, \& Adem, 2010), exponen que la idea central es usada como uno de los instrumentos de evaluación de la comprensión lectora ya que la misma implica una elaboración conceptual que denota jerarquización de información y relación entre conceptos relevantes presentes en la macro estructura del texto.

Para comprobar la comprensión lectora en los estudiantes de la Carrera de Inglés, se efectúan ejercicios de lectura recreativa con el fin de evaluar su apreciación sobre el tema leído, posteriormente se elaboran escritos, redacciones y ensayos sobre la temática impartida por el docente; sin embargo, la carencia de criterio argumentativo se evidencia en los trabajos que ellos realizan a diario, tales como el uso de conectores, el parafraseo de ideas relacionadas por los conectores, parafraseo de párrafos, la búsqueda de información relevante y en concordancia con la idea principal del texto.

La redacción requiere de expresiones entre conceptos detectados como más importantes en una oración que tenga coherencia y cohesión, desde el punto de vista composicional y semántico que permita ajustarse a la información presente en el texto leído. Por lo general, esta tarea demanda de la formulación de una oración compleja con un adecuado uso de conectores que manifiesten una correcta cohesión y relación de conceptos que respete lo que el autor desea interpretar en el texto fuente. La tarea implicaría un grado de comprensión global del texto fuente, que posibilite que el estudiante pueda distinguir la idea principal y las ideas secundarias y la relación existente entre ellas.

\section{Los conocimientos previos de los alumnos para la enseñanza y el aprendizaje del a redacción científica}

Los estudiantes de educación media, superior y los alumnos de otros niveles educativos asisten a las instituciones educativas con experiencias de vida, antecedentes escolares, formas de pensar y comprender la realidad; llegan con un conjunto de conocimientos previos que condicionan de manera importante las formas de aproximación hacia los contenidos.

Los conocimientos previos como instrumento para guiar la enseñanza, es un desafío constante para los docentes de los diferentes niveles educativos, los cuales pueden llegar a plantearse interrogantes tales como: ¿Por qué es necesario atender a los conocimientos previos de los alumnos?, ¿Cuáles son los conceptos que ellos poseen sobre los diferentes contenidos?, ¿Cómo desde su punto de vista los conceptos pueden llegar a fusionarse?

Para poder entender los cuestionamientos que los alumnos poseen desde el punto de vista de su aprendizaje familiar, escolar, media o superior de cómo fue influyendo el 


\section{Jornada de Investigación Universidad Tecnológica Empresarial de Guayaquil}

ámbito social, el entorno que lo rodea es mesurable que los docentes entienden la manera diversa que los estudiantes aprecian los contenidos aprendidos de diferentes asignaturas y la manera en que ellos asimilan un nuevo conocimiento.

Partiendo desde la perspectiva conductual del aprendizaje que tuvo su origen en la primera mitad del siglo pasado la particular manera en que los individuos se apropiaban de los conocimientos estaba basada en procesos de memorización y en formas mecánicas de fotografiar la realidad dentro de la mente. En este sentido el conocimiento es una copia fiel de la realidad y los alumnos pueden ser tratados como si su mente fueran páginas en blanco en las que la existencia de ideas propias es inconcebible sobre las cosas o conceptos que se les van a enseñar. Esta realidad que se vivió en el pasado ha venido presentando cambios, y ha dado paso a los nuevos enfoques educativos constructivistas que han ido tomando forma durante las últimas dos décadas del siglo pasado.

En este contexto se reconoce el papel activo de los alumnos en los procesos de apropiación y construcción del conocimiento, esto se ha concebido gracias a que el estudiante tiene interacción de los contenidos con la realidad, lo teórico y lo práctico; la manera diferentes que los sujetos van reconstruyendo, pensando, razonando y elaborando sus propias hipótesis; sobre cómo los objetos y las personas se relacionan entre sí y cómo son interpretadas estas interrogantes con los fenómenos que los rodean; así como la información y las explicaciones que otros les facilitan sobre la realidad; entonces se puede deducir que ningún aprendizaje inicia desde cero y que los seres humanos desde el momento de su concepción son personas activas e inteligentes que construyen y reconstruyen constantemente su conocimiento.

Una idea común entre docentes y elaboradores de materiales educativos es que la exploración de los conocimientos previos de los alumnos deben estar centradas en la recuperación de los contenidos escolares ya estudiados para ajustar y orientar la situación didáctica que van a implementar; sin embargo, la investigación sobre los procesos de aprendizaje ha mostrado que las experiencias que los individuos traen inciden en la apropiación de nuevos saberes tienen características muy variadas según la forma en que has sido aprehendidos y la manera en que organizan el pensamiento de los alumnos.

Desde el punto de vista de este aprendizaje significativo sería oportuno que los docentes utilizasen estrategias de aprendizajes basados en la construcción de nuevos conocimientos, en los cuales los individuos puedan fortalecer las destrezas y habilidades que necesitan para el desarrollar textos y se pueda evidenciar el sentido de su escritura, que los demás puedan entender cuál fue la idea principal que él quiso transmitir a sus lectores.

(Espino-Datsira, S, 1999) cita a Mariana Miras (1999) y sostiene El aprendizaje de un contenido es, en último término, el producto de una actividad mental constructiva que lleva a cabo el alumno (...) mediante la cual construye e incorpora a su estructura mental los significados y representaciones relativos al nuevo contenido, ahora bien, dicha actividad mental constructiva no puede llevarse a cabo en el vacío, partiendo de la nada la posibilidad de construir un nuevo significado, de asimilar un nuevo contenido; en definitiva, la posibilidad de aprender, para necesariamente por la posibilidad de "entrar en contacto" con el nuevo conocimiento.(pág. 22).

La necesidad de considerar los conocimientos previos como punto de partida para la 


\section{Jornada de Investigación Universidad Tecnológica Empresarial de Guayaquil}

adquisición de nuevos aprendizajes encuentra su justificación en la propia definición constructivista del aprendizaje. Desde esta perspectiva, se entiende que el aprendizaje de un nuevo contenido es, en última instancia, el producto de una actividad mental mediante en la cual se construye y se incorpora a la propia estructura mental de los significados y representaciones relativos al nuevo contenido. Pero, esta actividad mental constructiva no puede realizarse en el vacío, partiendo de la nada, sino que la posibilidad de construir un nuevo significado, de asimilar un nuevo contenido, en definitiva, la posibilidad de aprender, pasa inexcusablemente por la necesidad de entrar en contacto con el nuevo conocimiento.

Partiendo de esta premisa se considera entonces que los alumnos que leen sus escritos sobre la importancia de la investigación en la vida cotidiana, manifiestan ideas pocos precisas sobre la investigación y cuál es su relación con la ciencia, tecnología y el aprendizaje, una tesis oscura se encuentran presente en la interpretación personal que él realiza, utilizando términos poco claros para desarrollar su apreciación sobre los contenidos que pretende demostrar.

Entonces es necesario manifestar que los procesos cognitivos de los alumnos están en la base de sus conocimientos previos, son construcciones personales que se han formado a lo larga historia, en gran medida han sido elaboradas de manera espontánea en su interacción cotidiana con el mundo y las personas por los que son previos a la instrucción, en este sentido reflejan las ideas que se han formado por sí mismos a través de inferencias y suposiciones sobre los datos que reciben del entorno; es decir, los saberes y las prácticas sociales influyen de manera importante en conceptos y la forma de comprender la realidad y actuar en el mundo utilizando sus valores y representaciones simbólicas.

\section{Materiales y métodos}

El estudio se llevó a cabo con un enfoque cualitativo, se elaboró como método empírico un test de diagnóstico dirigido a 35 estudiantes del quinto, sexto y séptimo semestre de la Carrera de Inglés de la Facultad de Educación, para descubrir el nivel de comprensión de lectura, en el primer ítem se contempla un fragmento de texto con el objetivo de diagnosticar si los estudiantes pueden diferenciar y discriminar los tipos de texto de manera narrativa, literaria, científica y humanística. En el segundo ítem se pide a los estudiantes que ubiquen los signos de puntuación de manera correcta. En el tercer ítem se refleja un párrafo donde se debe identificar y encerrar los verbos y en último indicador consiste en leer un fragmento de la lectura y extraer con sus propias palabras la idea principal y las ideas secundarias en el texto.

\section{La Evaluación}

Para medir los resultados de la evaluación es importante determinar el rendimiento de los estudiantes hacia el desarrollo de una escritura comprensiva, la cual han sido elaborados con el objetivo de perfeccionar en los alumnos las destrezas para descubrir detalles implícitos, jerarquizar datos, identificar la idea central con las ideas secundarias, descubrir personajes principales y secundarios, realizar cuadros comparativos, lluvia de ideas, utilizar la crítica investigativa, establecer causas y efectos sobre el contenido implícito y explícito. Estas instrucciones plantean diversos tipos de enseñanza con los cuales los estudiantes descubren el interés por la lectura y los beneficios que trae consigo, convirtiéndose en un aprendizaje divertido y entretenido.

Cabe señalar que la función de evaluación consistirá en dar conocer a los estudiantes 
Revista Ciencia \& Tecnología No. 16, 31 de octubre de 2017 ISSN impreso: 1390 - 6321

\section{Jornada de Investigación Universidad Tecnológica Empresarial de Guayaquil}

los problemas académicos a priori; para concientizar en ellos la mejora continua, la constante investigación y actualización de contenidos, de esta manera poder optimizar los resultados y lograr alcanzar los objetivos que se desea obtener. Para este propósito se ha utilizado la rúbrica como una de las técnicas aplicadas en la muestra de estudiantes la misma que fue desarrollada como una plantilla la cual contiene y concreta los criterios de evaluación (Del Campo Moreno, \& Esteban, 2011) (Véase tabla 1).

Tabla 1. Criterios de Evaluación

\begin{tabular}{|c|c|c|c|}
\hline Cnterios & $\begin{array}{l}\text { Excelente } \\
\qquad 100 \%\end{array}$ & $\begin{array}{l}\text { Bueno } \\
80 \%-70 \%\end{array}$ & $\begin{array}{l}\text { Regular } \\
60 \% \text { o menos }\end{array}$ \\
\hline $\begin{array}{l}\text { Dominio } \\
\text { semantico }\end{array}$ & $\begin{array}{l}\text { Escribe oraciones y } \\
\text { párrafos entrelazando } \\
\text { oraciones con sentido } \\
\text { completo y autonomia } \\
\text { sintáctica, tomando en } \\
\text { cuenta que los escritos } \\
\text { tengan coherencia y } \\
\text { cohesión. }\end{array}$ & $\begin{array}{l}\text { Escribe oraciones y párrafos } \\
\text { entrelazando oraciones con } \\
\text { sentido completo y } \\
\text { autonomia sintáctica. }\end{array}$ & $\begin{array}{l}\text { No redacta oraciones ni } \\
\text { párrafos que estén } \\
\text { erilazados con sentido } \\
\text { completo y autonomía } \\
\text { sintáctica. }\end{array}$ \\
\hline $\begin{array}{l}\text { Identitica el } \\
\text { contenido de } \\
\text { un texto }\end{array}$ & $\begin{array}{l}\text { Realiza discriminaciones } \\
\text { acordes a las ideas que el } \\
\text { autor pretende trasmitir a } \\
\text { sus lectores y escribe de } \\
\text { forma jerarquizada los } \\
\text { argumentos que ha } \\
\text { logrado comprender }\end{array}$ & $\begin{array}{l}\text { Realiza discriminaciones } \\
\text { acordes a las ideas que el } \\
\text { autor pretende trasmitir a sus } \\
\text { lectores y escribe de forma } \\
\text { organizada. }\end{array}$ & $\begin{array}{l}\text { Realiza discriminaciones } \\
\text { acordes a las ideas que el } \\
\text { autor pretende trasmitir a } \\
\text { sus lectores no realiza } \\
\text { escritura organizada }\end{array}$ \\
\hline $\begin{array}{l}\text { Interpreta } \\
\text { adecuadame } \\
\text { nte los tipos } \\
\text { de texto. }\end{array}$ & $\begin{array}{l}\text { Infiere con sentido según } \\
\text { el proceso que va } \\
\text { estableciendo de acuerdo } \\
\text { a la información implicita } \\
\text { en el texto, siendo } \\
\text { coherente con la temática } \\
\text { establecida. }\end{array}$ & $\begin{array}{l}\text { Infiere con sentido según el } \\
\text { proceso que va } \\
\text { estableciendo de acuerdo a } \\
\text { la información implicita y } \\
\text { explicita en el texto. }\end{array}$ & $\begin{array}{l}\text { Infiere con poco sentido } \\
\text { el proceso que va } \\
\text { estableciendo do de } \\
\text { acuerdo a la intormación } \\
\text { implicita en el texto. }\end{array}$ \\
\hline $\begin{array}{l}\text { Material de } \\
\text { apoyo }\end{array}$ & $\begin{array}{l}\text { Utiliza libros de la } \\
\text { biblioteca local para } \\
\text { afianzar los conocimientos } \\
\text { en base al tema en } \\
\text { desarrollo, comprende, } \\
\text { critica e interpreta } \\
\text { asertivamente. }\end{array}$ & $\begin{array}{l}\text { Utiliza libros de la biblioteca } \\
\text { Iocal con la finalidad de } \\
\text { consolidar los conocimientos } \\
\text { en base al tema en } \\
\text { desarrollo. }\end{array}$ & $\begin{array}{l}\text { No utiliza libros de la } \\
\text { biblioteca local para } \\
\text { afianzar ras los } \\
\text { conocimientos en base al } \\
\text { tema en desarrollo. }\end{array}$ \\
\hline $\begin{array}{l}\text { Utiliza } \\
\text { fuentes } \\
\text { bibliográfica } \\
\text { s }\end{array}$ & $\begin{array}{l}\text { Las fuentes citadas son } \\
\text { de calidad y fidedignas } \\
\text { reconoce las bases de } \\
\text { datos autorizadas yy } \\
\text { discrimina las } \\
\text { depredadores, maneja } \\
\text { correctamente las norma } \\
\text { APA 6ta. }\end{array}$ & $\begin{array}{l}\text { Las fuentes citadas son de } \\
\text { calidad y fidedignas } \\
\text { reconoce las bases de datos } \\
\text { autorizadas y discrimina las } \\
\text { depredadores, las normas } \\
\text { regularmente las notiza } \\
\text { APA 6ta. }\end{array}$ & $\begin{array}{l}\text { Las fuentes citadas } \\
\text { carecen de información } \\
\text { de calidad y no reconoce } \\
\text { las bases de datos } \\
\text { autonzadas no maneja las } \\
\text { norma APA } 6 \text { ta. }\end{array}$ \\
\hline
\end{tabular}

Fuente: Elaboración propia

\section{Resultados}

En este apartado se detallan los resultados que se obtuvieron a través de la aplicación del test de diagnóstico que se utilizó para medir el conocimiento de la escritura científica y cuyo medio de evaluación fue la rúbrica, esto se realizó con el afán de identificar los criterios de evaluación, para lo cual se ha utilizado un total de 35 estudiantes voluntarios: 5 estudiantes en el quinto semestre, 15 en el sexto y 15 en séptimo de la Carrera de Inglés. La estadística descriptiva de la muestra indica que la mayor parte de los estudiantes $(57 \%)$ tienen problemas de dominio semántico y sintáctico; es decir, no escriben oraciones de manera organizada ni párrafos 


\section{Jornada de Investigación Universidad Tecnológica Empresarial de Guayaquil}

entrelazados que contengan coherencia y cohesión y den a entender cualquier información que desean trasmitir. El orden de las palabras y oraciones pasa a segundo plano en la redacción, así mismo se observa cierto grado de dificultad que los estudiantes tienen para construir textos más complejos, haciendo uso de los conectores adecuados en tiempo, espacio, género y número y expresarlos de una forma en la que todos puedan comprender.

En el segundo criterio de evaluación se descubre que el $60 \%$ de los estudiantes realizan discriminaciones poco coherentes y no acordes a las ideas que el autor pretende comunicar, la escritura utilizada carece de organización semántica y la redacción es incomprensible para los lectores. La deficiencia para reconocer verbos simples como el de descripción se observa en los mensajes que desea transmitir, estos muchas veces son confundidos con adjetivos, adverbios y otros suelen ser reemplazados con un sustantivo. Otra de las causas más relevantes es que a los estudiantes se les dificulta identificar la idea principal, lo cual es indispensable para dar inicio a una lectura crítica y comprensiva. El (37\%) acierta de manera favorable en la lectura que se ha seleccionado para evaluar este criterio; sim embargo, cabe recalcar que solamente el (3\%) de los estudiantes con los cuales se ha realizado este estudio dominan la lectura y lo aplican al momento de realizar la escritura.

Por otra parte, en el tercer criterio se basa en la interpretación de lectura, muestra que el $(69 \%)$ de los estudiantes se les dificulta reconocer las diferencias que existen entre la información que está presente en los diferentes tipos de texto; mientras que el $(23 \%)$ pueden realizar discriminaciones acordes a las ideas que el autor pretende trasmitir a sus lectores. Con el afán de que los estudiantes puedan fortalecer las habilidades escritas, sería conveniente que los docentes implementen técnicas y métodos que ayuden a desarrollar las destrezas hacia la lectura y estar orientadas a una mejor percepción en el reconocimiento de las ideas principales que el texto desea trasmitir, los cual llevará a estructurar y mejorar la redacción de textos con diferentes enfoques semánticos tales como: narrativo, argumentativo, descriptivo, expositivo y científico.

Para los estudiantes de la Carrera de Inglés el uso de los libros de la biblioteca local no ha sido aprovechado en su totalidad; esto se debe a que solo se limitan a escuchar las clases que el docente imparte en cada asignatura y no sienten el interés para desarrollar el tema tratado, tampoco a investigar de forma consciente las tareas autónomas que sirven de fortalecimiento para el desarrollo cognitivo. Otra de las causas que podrían involucrarse a este criterio es que muchos de los docentes carecen de iniciativa al momento de impartir los conocimientos y el costo para incentivar hacia la una investigación científica con responsabilidad y criterio formado suele ser decadente, lo que irrelevantemente no suele fomentar el interés hacia la búsqueda de información en diferentes recursos bibliográficos para enriquecer sus conocimientos.

El último criterio radica en que la información que los estudiantes proveen es de baja calidad y contenido científico, el copiar y pegar forma para de su vida, es como un tatuaje imborrable en el cuerpo, los derechos del autor están ubicados en el inconsciente del estudiante y los esfuerzos de algunos docentes por borrar estas costumbres impregnadas en los alumnos no han sido logradas, los reglamentos que las normas APA 6ta dictan no están en su vocabulario investigativo. (Cuadro 2). 
Revista Ciencia \& Tecnología No. 16, 31 de octubre de 2017 ISSN impreso: 1390 - 6321

\section{Jornada de Investigación Universidad Tecnológica Empresarial de Guayaquil}

Tabla 2. Resultados en Porcentajes de Criterios de evaluación

\begin{tabular}{|l|r|r|r|r|}
\hline \multirow{2}{*}{$\begin{array}{c}\text { Resultado de la Rúbrica aplicado a } \\
\text { estudiantes }\end{array}$} & \multicolumn{5}{c|}{ PORCENTAJES } \\
\cline { 2 - 6 } & Excelente & Bueno & Regular & total \\
\hline Dominio semántico & $9 \%$ & $34 \%$ & $57 \%$ & $100 \%$ \\
\hline $\begin{array}{l}\text { Identifica el contenido de un texto } \\
\text { Interpreta adecuadamente los tipos de }\end{array}$ & $3 \%$ & $37 \%$ & $60 \%$ & $100 \%$ \\
texto. & $9 \%$ & $23 \%$ & $69 \%$ & $100 \%$ \\
\hline $\begin{array}{l}\text { Material de apoyo } \\
\text { Utiliza fuentes bibliográficas }\end{array}$ & $14 \%$ & $43 \%$ & $43 \%$ & $100 \%$ \\
\hline \multicolumn{4}{|c|}{ Número de estudiantes evaluados } \\
\hline
\end{tabular}

Fuente: Elaboración propia

En el siguiente gráfico de línea se evidencia los resultados en porcentaje de los Criterios de Evaluación que se aplicaron en este estudio de Escritura semántica creciente y decreciente; en la cual se muestra las diferentes variantes evaluadas desde el grado de excelencia hasta regular. En este caso la línea gris expresa el alto porcentaje de deficiencia que tienen los estudiantes para realizar escritos, ensayos o artículos científicos con sentido coherente y aplicando la introducción el desarrollo y la conclusión. Por otra parte, existe un promedio de concordancia e igualdad en el uso de material de apoyo, debido a que la mitad de los estudiantes evaluados recurren a las bibliotecas con el afán de fortalecer y sustentar sus investigaciones. Mientras que los demás muestran cierto desinterés y desmotivación hacia la investigación científica. (Véase gráfico 1 ).

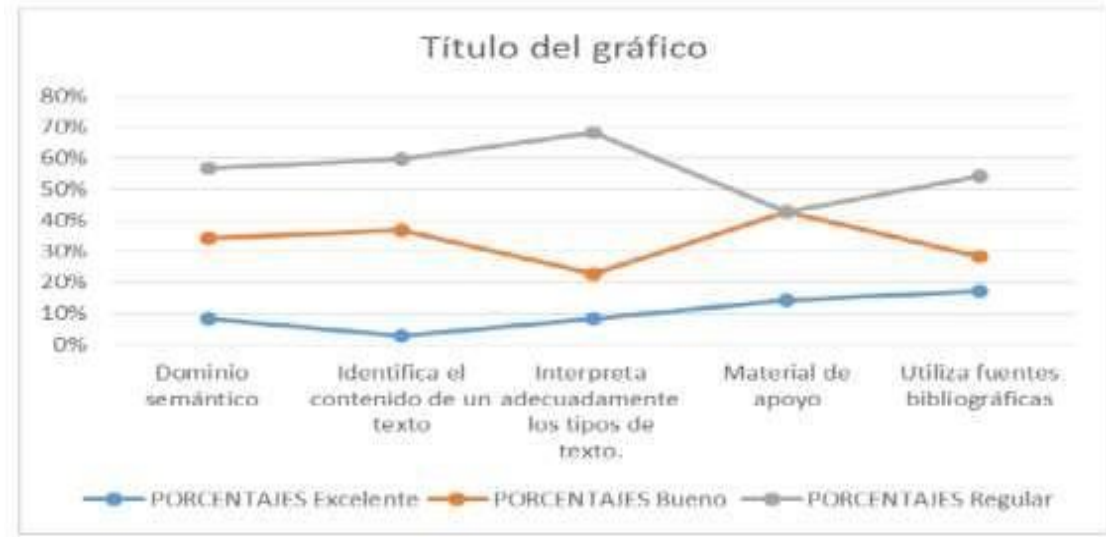

Gráfico 1 Investigación científica

Fuente: elaboración propia

\section{Conclusiones}

La expresión morfológica a la deriva lo que hace es agigantar la brecha en la escritura comprensiva, la redacción requiere de expresiones entre conceptos detectados como más importantes en una oración que tenga coherencia y cohesión, desde el punto de vista composicional y semántico ye permita ajustarse a la información presente en el texto leído. Una vez que los estudiantes dominen cognitiva y operacionalmente los segmentos idiomáticos podrían utilizar las preposiciones y conjunciones para armar párrafos que complementen su estructura morfológica. 


\section{Jornada de Investigación Universidad Tecnológica Empresarial de Guayaquil}

El alto porcentaje de deficiencia que tienen los estudiantes para realizar escritos, ensayos o artículos científicos con sentido coherente y aplicando la introducción el desarrollo y la conclusión. Por otra parte, existe un promedio de concordancia e igualdad en el uso de material de apoyo, debido a que la mitad de los estudiantes evaluados recurren a las bibliotecas con el afán de fortalecer y sustentar sus investigaciones. Mientras que los demás muestran cierto desinterés y desmotivación hacia la investigación científica.

La exploración de los conocimientos previos de los alumnos debe estar centradas en la recuperación de los contenidos escolares ya estudiados para ajustar y orientar la situación didáctica que van a implementar; desde el punto de vista de este aprendizaje significativo sería oportuno que los docentes utilizasen estrategias de aprendizajes basados en la construcción de nuevos conocimientos, en los cuales los individuos puedan fortalecer las destrezas y habilidades que necesitan para el desarrollar textos y se pueda evidenciar el sentido de su escritura, que los demás puedan entender cuál fue la idea principal que él quiso transmitir a sus lectores.

Los resultados obtenidos en base al problema detectado en las destrezas y habilidades de los estudiantes para la redacción de ensayos y artículos científicos avizoran la posibilidad de buscar alternativas metodológicas que implican hacia el mejoramiento de sus escrituras mediante un dominio semántico que haga posible la distribución del proceso comprensivo, para la utilización correcta de signos.

\section{Referencias bibliográficas}

Asamblea Nacional (2008). Constitución de la República del Ecuador. Montecristi. Registro Oficial \# 449.

Del Campo Moreno, P., \& Esteban, L. P. (diciembre de 2011). La Webquest como estrategia metodológica en la enseñanza universitaria de la asignatura de Contabilidad de Empresas Turísticas. EDUTEC. Revista electrónica de tecnología educativa, 38.

El Mercurio. (16 de 01 de 2015). Escritura falla más entre secundarios, dice estudio. Además, Senescyt detectó que $30 \%$ de estudiantes de posgrado no culmina por problemas al escribir., pág. 12.

Espino-Datsira, S. (1999). La toma de apuntes. Visión de los estudiantes universitarios mexicanos. Iberoamericana de Educación Superior, 22.

Feldman, S., Anderson, V., y Mangurian, L. (2001). Teaching effective scientific writing. Journal of College Science Teaching, 30 (7), 446-450.

Insirillo, P., González, M. S., \& Adem, A. (2010). La redacción de ideas principales de textos académicos en inglés: sus beneficios y dificultades como instrumento de evaluación de la lecto comprensión. En Castel y Cubo (editores), La renovación de la palabra en el bicentenario de la Argentina, 661-679.

Searle, J. R. (2014). What Your Computer Can't Know. New York: The New York Review of Books. 